\title{
La Brecha Digital de Género como factor limitante del desarrollo femenino
}

\author{
Digital gender gap as a limiting factor for female development
}

Silvia C. Acosta-Velázquez ${ }^{a}$,Elba M. Pedraza-Amador ${ }^{b}$

\begin{abstract}
:
The development of new Information and Communication Technologies (ICT), whose history starts from the 70's, has a strong impact on today's society due to the increasing flow of information and its management in different areas, as well as its applications in the organizational environment, where it is related to the automation of processes, management control and the exchange of scientific information.

ICT help communication, transmission of information and work, and contribute to improving the quality of life of people, however, with the arrival of the Era of the Internet, Information and Knowledge, new skills and competencies are required to interact and communicate, to use digital devices, to access information and manage it, which generates new inequalities and forms of exclusion, due to access to the development of digital skills is based on academic training, socioeconomic status, age and gender, whereby women have the least opportunities.

The digital gender gap is evident and both, in the formal labour field and in the implementation of entrepreneurial projects, women often face a series of obstacles to enter the business world, based on their digital skills. For this reason, this document focuses on the review of the particularities of the digital divide and its variables, as well as their possible solutions, with the purpose of obtaining information that in the medium term allows to design a training proposal for women entrepreneurs in the state of Hidalgo, which contributes to counteract this situation of inequality and exclusion in the workplace.
\end{abstract}

Keywords:

Female entrepreneurship, Digital gap, technology

\section{Resumen:}

El desarrollo de nuevas Tecnologías de Información y Comunicación (TIC), cuya historia inicia a partir de la década de los 70’s, tiene un fuerte impacto en la sociedad actual en función del creciente flujo de información y su manejo en los diferentes ámbitos, así como sus aplicaciones en el entorno organizacional, en donde se asocia a la automatización de los procesos, el control de la gestión y el intercambio de información científica.

Las TIC facilitan la comunicación, la transmisión de información y el trabajo, y contribuyen a mejorar la calidad de vida de las personas, sin embargo, con la llegada de la Era del Internet, la Información y el Conocimiento, se requieren nuevas habilidades y competencias para interactuar y comunicarse, hacer uso de dispositivos digitales, acceder a la información y gestionarla, lo cual genera nuevas desigualdades y formas de exclusión debido a que el acceso para el desarrollo de competencias digitales se encuentra en función de la formación académica, el nivel socioeconómico, la edad y el género, por lo que las mujeres son quienes menores oportunidades tienen.

La brecha digital de género es evidente y tanto en el ámbito laboral formal como en la puesta en marcha de proyectos emprendedores, las mujeres suelen enfrentar una serie de obstáculos para incorporarse al mundo de los negocios en función de sus habilidades digitales, por tal motivo, el presente documento se centra en la revisión de las particularidades de la brecha digital y sus variables, así como de sus posibles soluciones, con el propósito de obtener información que en el mediano plazo permita plantear una propuesta formativa para emprendedoras en el estado de Hidalgo, que contribuya a contrarrestar esta situación de desigualdad y exclusión en el ámbito laboral.

Palabras Clave:

Emprendimiento femenino, brecha digital, tecnología

\section{Introducción}

Uno de los factores que en la actualidad tiene mayor impacto en el ámbito de los negocios es la tecnología, cuyos desarrollos contribuyen a

\footnotetext{
${ }^{a}$ Autor de Correspondencia. Universidad Autónoma del Estado de Hidalgo, Escuela Superior de Tizayuca, https://orcid.org/0000-0001-89727748, Email: silvia_acosta2066@uaeh.edu.mx

b Universidad Autónoma del Estado de Hidalgo, Escuela Superior de Tizayuca, https://orcid.org/0000-0002-7182-2437, Email: elbam@uaeh.edu.mx
} 
generar mayor eficiencia y productividad en las empresas y favorecen los emprendimientos, al ser una herramienta a través de la cual los emprendedores logran acceder a una gran cantidad de información sobre el mercado, sus clientes y la competencia, visibilizan sus proyectos y llegan a un mayor número de personas.

En una sociedad caracterizada por los avances tecnológicos y prácticas laborales relacionadas directamente al uso de internet, el acceso al empleo está condicionado al dominio de competencias y habilidades para uso y aplicación eficiente de Tecnologías de la Información y la Comunicación (TIC). La introducción de nuevas tecnologías favorece una mayor productividad de las empresas, sin embargo, modifica los escenarios laborales al reemplazar funciones humanas en tanto que genera "nuevos requerimientos y exigencias tecnológicas" (Aguilar, 2011, p.34) que ponen en juego la estabilidad de los trabajadores, ante el riesgo de perder sus puestos de trabajo.

En respuesta a estas nuevas necesidades, las Universidades han incorporado el desarrollo de competencias digitales en los programas para formar nuevos profesionistas, capacitándolos para resolver problemas y enfrentar los actuales desafíos del mundo de los negocios. Sin embargo, dada la desigualdad que existe entre hombres y mujeres en todos los ámbitos de la sociedad y en todos los lugares, las niñas y mujeres jóvenes tienen menores oportunidades que los hombres para acceder a la educación (Bonder, 2002), incluso la educación de las mujeres fue objeto de debate en Hispanoamérica durante todo el siglo XIX y hasta bien avanzado el XX (Barrios \& Manyoma, 2019) limitando su participación al trabajo no remunerado, el cuidado del hogar, y en el mejor de los casos al desarrollo de proyectos emprendedores con escasas posibilidades de consolidación.

En relación a las actividades emprendedoras, estudios recientes (Informe GEM, España 2015) muestran una baja participación femenina, atribuible a barreras sociales y a prejuicios en relación a sus habilidades y capacidad para el desempeño de actividades profesionales (Kantor, 2002 en Sabater, 2018), en tanto que en México, de acuerdo a cifras de la OCDE en un estudio sobre las brechas de género, la minoría femenina en proyectos de emprendimiento está relacionada a factores entre los que destacan escasa disponibilidad de recursos financieros, falta de acceso a la educación y estigmas sociales.
No obstante, son precisamente esas barreras las que llevan a las mujeres a generar sus emprendimientos, como respuesta a la falta de valoración en el empleo y la dificultad de acceder a formación profesional en ciertas disciplinas (Viadana, Zubeldía, Tomarelli \& Morales, 2016).

\section{TIC: efectos positivos y adversos}

En la década de los ochentas, el mundo se transformó con la explosión de TIC en los ámbitos económico y social. Los usuarios de distintos campos -incluyendo la ciencia, el comercio y la educación- debieron hacer uso de ellas sin conocimiento previo de su naturaleza, lo que dio lugar a un acelerado proceso de alfabetización digital que no siempre fue equitativo y dejó en la marginación a quienes no las utilizan de manera natural. En este sentido, Cabrera (2005) refiere al componente tecnológico, concretamente a las TIC como un factor generador de exclusión que frena la incorporación de las personas a la sociedad en función de su situación económica y de su género, enfatizando que existe una "desigualdad de género que hace que las mujeres accedan en menor medida y para usos diferentes a las TIC" (p. 14).

Al respecto Arenas (2011) señala que no todos los ciudadanos tienen acceso a las nuevas tecnologías ni hacen uso de ellas en condiciones de igualdad, y qué en un futuro, los individuos que no estén calificados tendrán mayores probabilidades de ser marginados, destacando que el género femenino es el más perjudicado.

Por otra parte, la encuesta realizada en enero 2000 por Mujeres Latinas en Internet señala que únicamente cierto tipo de mujeres tienen acceso a estas herramientas, predominando las que cuentan con estudios superiores, de edades entre 22 y 35 años, sin embargo, refiere que el uso principal no es para situaciones laborales, cuestionándose entonces si el acceso a las nuevas tecnologías abre para ellas nuevas oportunidades de capacitación y empleo, como el teletrabajo, o únicamente contribuye a mejorar su posición social (Bonder, 2002).

Es así entonces que a pesar de que las TIC impactan positivamente a todos los campos del saber y del hacer humano y de alguna manera flexibilizan la vida laboral de las mujeres, haciéndola compatible con su vida familiar y personal, plantean limitantes para el desarrollo profesional y crecimiento de individuos con baja calificación en competencias digitales, a quienes se degrada a puestos de trabajo donde prevalece la sobreexplotación y los horarios extendidos e invasivos a su vida privada.

Debe tenerse en cuenta también que las nuevas tecnologías de información y comunicación son sólo un instrumento cuyos efectos, positivos o adversos se 
encuentran en función del uso que se les da, sin embargo, ante la evidencia del mayor dominio masculino en su utilización y manejo, es conveniente considerar la "brecha digital" que la desigualdad para acceder a ellas ha provocado (Arenas, 2011)

\section{Brecha Digital y sus variables}

La economía y la sociedad contemporáneas se han visto transformadas por el desarrollo de las TIC, que dan lugar a nuevas formas de organización de la producción y del trabajo en las empresas (Lera, Hernández \& Blanco, 2003), contribuyen al logro de los objetivos organizacionales y personales y modifican los procesos de "adquisición, almacenamiento, evaluación, transmisión, distribución y diseminación de la información" (Mendoza \& Caldera, 2014, p. 126) mejorando la comunicación.

Los efectos positivos de su desarrollo, evolución e implantación en las empresas son cada vez más evidentes, sin embargo, también se manifiestan los desafíos que enfrentan los individuos para su acceso y aprovechamiento, refiriendo una distancia tecnológica - denominada "brecha digital"- en el acceso a la información y a las TIC, así como en el uso de Internet, que limita la interacción, la integración social y las oportunidades de empleo a las generaciones jóvenes y al género femenino.

Esta brecha digital que deja a una parte de la sociedad al margen de las nuevas tecnologías es generada principalmente por factores socioeconómicos, geográficos 0 demográficos y crea una nueva desigualdad (Alva, 2014) que el sistema educativo ha intentado minimizar mediante la alfabetización digital en las escuelas y la creación de infraestructura, con el propósito de facilitar el acceso a ellas.

Aguinaga (2016) destaca que la mayor brecha se encuentra entre los que acceden y los que no acceden a las TIC, y su medida cambia en función del incremento al uso y el consumo; así mismo Toudert (2019), refiere que más allá del acceso, la brecha digital depende de las habilidades y el aprovechamiento de los usuarios de internet, en concordancia con Castaño (2009), quien señala que la mayor barrera no es el acceso sino el uso, toda vez que las oportunidades que las nuevas tecnologías crean para el desarrollo profesional de los individuos depende en gran medida de la forma en que las utilizan.

Aunque de manera general la brecha digital se relaciona con el uso de internet, Varela (2015) enuncia otras variables generadoras de exclusión digital con base a un estudio de la población española, que refiere no es de dimensión reducida, lo que la exime de la sujeción exclusiva a la población de dicho país; abarca todos los ámbitos de la sociedad y sus efectos se extienden más allá de un territorio determinado.

Con el propósito de exponer las principales variables de la exclusión digital, clasifica la brecha digital en las modalidades de:

a) cuestión de género,

b) cuestión de edad,

c) de índole formativa, identificada como analfabetismo tecnológico,

d) de índole funcional, atribuible a algún tipo de discapacidad que imposibilita el acceso,

e) de índole económica o imposibilidad de asumir los costos del servicio

f) de índole geográfica o territorial, caracterizada por la ausencia de infraestructura en un determinado lugar,

g) urbana-rural, que agrupa aspectos económicos y territoriales, y

h) de índole formativa-laboral (Varela, 2015, p. 31).

Sobre este último aspecto, Aguinaga (2016) enfatiza "el acceso al estudio y la ocupación laboral en las empresas tecnológicas" (p. 19) como uno de los factores principales de diferencias de género entre jóvenes, y señala la aparición de una nueva brecha a partir de quienes no participan en las TIC y por consiguiente, no son beneficiarios de la información a que se tiene con dichas herramientas.

Como se observa, las desigualdades en función de conocimientos sobre TIC, habilidades para su acceso, habilidades para el desempeño laboral en entornos relacionados a ellas (Gil-Juarez, Vitores, Feliu \& Vallllovera, 2011) están presentes, dejando en claro que las brechas abiertas a partir del acceso y aprovechamiento de TIC y la escasa participación de mujeres en empleos relacionados con tecnologías no hacen otra cosa que confirmar la discriminación de que es objeto la mujer en los ámbitos laboral y social (Romero \& Varela, 2018); a pesar de que el discurso general gire en torno a la igualdad de acceso para hombres y mujeres, las cifras a nivel mundial refieren severas disparidades, que conducen a la reflexión sobre la brecha digital por cuestiones de género.

\section{Brecha digital de Género}

Definida como la diferencia entre las tasas masculina y femenina de una variable, la brecha de género destaca las desigualdades en cualquier ámbito, en todos los contextos.

En cuanto a la incorporación a las nuevas tecnologías y el acceso a internet, ser hombre o mujer es un hecho determinante de la brecha digital de género (Varela, 2015), que de acuerdo a lo expresado por Gil-Juarez, Vitores, Feliu \& Vall-Ilovera, (2011) no es un problema derivado exclusivamente del acceso y uso de las TIC, 
sino que tiene que ver también con la formación universitaria, debido a la amplia desventaja femenina en relación al dominio masculino, en estudios relacionados al ámbito tecnológico.

Esta exclusión de las mujeres en espacios relacionados con educación y empleo en áreas de ciencia y tecnología lesiona sus derechos fundamentales y las sujeta a una serie de prescripciones sociales y normativas (Arenas, 2011) a pesar de las consideraciones sobre la igualdad de oportunidades para el empleo y la ocupación laboral.

De acuerdo a datos de la Organización Internacional del Trabajo (OIT), los hombres adquieren competencias tecnológicas en mayor proporción que las mujeres, quienes enfrentan una gran cantidad de barreras que impiden su acceso a nuevas oportunidades laborales más productivas y de mayor valor agregado, así mismo, aunque se reporta un incremento en la cantidad de mujeres inscritas en los diferentes niveles educativos, los resultados apuntan a una baja inclusión en los estudios relacionados con ciencia y tecnología, y por tanto, una escasa representación de fuerza laboral en dichos ámbitos (OIT, 2019).

Esta misma información fue revelada en el informe de la Organización para la Cooperación y el Desarrollo Económicos (OCDE, 2008), en el que se señala la baja representación de mujeres en dichas áreas, en los países miembros de esta organización, aludiendo más a cuestiones de actitud que de aptitudes. Un dato importante presente en este informe refiere el acceso a las TIC en los puestos de trabajo, destacando que aun cuando hay mujeres que han obtenido títulos universitarios en las áreas mencionadas, su participación laboral se limita a puestos de baja importancia en donde desempeñan tareas rutinarias que requieren un escaso nivel de competencias digitales, evidenciando una vez más, la discriminación laboral de que se hace objeto al género femenino.

En relación a los emprendimientos liderados por mujeres, la OCDE señala los estigmas culturales, el bajo nivel educativo, falta de experiencia laboral, escasa o nula habilidad para el manejo de TIC, y discriminación de género como una de principales limitaciones que enfrentan las mujeres (Garcia, 2019) al emprender sus negocios, que por lo general son una "extensión" de sus actividades hogareñas, están ubicados en subsectores con bajo valor agregado y tienen una escasa utilización de tecnología, ya sea porque no están preparadas para desarrollar un negocio, o bien por falta de recursos y de capacitación para su acceso, utilización y manejo.

Lo anterior es sólo uno de tantos ejemplos de las limitaciones que las normas culturales, sociales y laborales imponen a las mujeres para acceder a los mercados de trabajo, los espacios públicos, la tecnología y la información (Aguinaga, 2016), evidenciando la amplitud de la brecha que expone las diferencias entre ambos géneros.

\section{Hacia la reducción de la brecha digital de género}

La brecha digital de género no es un proceso natural ni es producto de una elección libre, sino el resultado de una construcción social fundamentada en expectativas y restricciones relacionadas con la edad, la educación, el rol histórico y cultural conferido a las mujeres y otros prejuicios y estereotipos, por tanto, debe ser entendida de forma diferente y resolverse mediante políticas específicas (Castaño, 2009) para la organización del trabajo y los horarios, los sistemas de acceso y promoción y la eliminación de la cultura empresarial predominantemente masculina.

Si bien es cierto que las mujeres se han incorporado paulatinamente al ámbito de los negocios adaptando sus necesidades personales a sus proyectos de emprendimiento, su inclusión en los procesos de apropiación de la tecnología y la superación de las barreras culturales, sociales y laborales implica un gran reto. No resulta suficiente la incorporación de prácticas educativas tendientes a desarrollar las competencias digitales en la población de jóvenes, se precisa de la incorporación de valores que promuevan la igualdad de género y coadyuvar el cambio de la cultura laboral, orientándolo hacia el incremento de presencia femenina en puestos que impliquen responsabilidad y participación en la sociedad de la información para alcanzar las metas propuestas.

Tomando como base las barreras de acceso a que se enfrentan las mujeres, los estereotipos masculinos, las restricciones académicas y laborales y la ausencia de modelos femeninos que constituyan un referente en este ámbito, en los últimos veinticinco años alrededor del mundo se han llevado a cabo diversas intervenciones con la participación de instituciones educativas, organizaciones empresariales, asociaciones independientes y gobiernos que a través de talleres, cursos, plataformas de apoyo, campañas y grupos de trabajo, entre otras alternativas, tienen el propósito de incrementar el número de mujeres usuarias de TIC (Gil-Juarez, Vitores, Feliu \& Vallllovera, 2011) que, sin embargo, no han logrado un cambio en las tendencias discriminatorias, lo que permite suponer que la brecha digital de género es un problema más complejo, que requiere ser abordado no desde los factores que propician la exclusión sino a partir de aquellos que fomentan la participación de las mujeres en el acceso y utilización de las TIC y el internet. 


\section{Conclusiones}

Hacer referencia a la desigualdad que existe entre hombres y mujeres para el acceso a las TIC y a la sociedad de la información implica profundizar en la "brecha digital de género" y reflexionar acerca de la inferioridad del género femenino en cuanto a oportunidades de educación y empleo, no únicamente en las áreas que involucran responsabilidad y habilidades digitales, sino de manera general, a pesar de las consideraciones de igualdad presentes en la sociedad actual.

Desde nuestra perspectiva, la reducción de la brecha digital de género es una tarea compleja que requiere la participación del sistema educativo, el gobierno y la sociedad en general, en tanto que implica la eliminación de los roles de género, la inclusión de programas de alfabetización digital en las escuelas y realizar acciones concretas que hagan un llamado a la conciencia social.

Pese a que la superación de la brecha digital de género esté todavía lejana, y que es uno de los principales factores que obstaculizan la inserción de las mujeres al ámbito laboral, las desigualdades existentes se han convertido en una motivación para el desarrollo de proyectos emprendedores liderados por representantes del género femenino, con cada vez mayores posibilidades de reconocimiento y generación de utilidades.

Considerando que las habilidades para el uso y manejo de TIC permiten a las personas el acceso a mayores oportunidades de empleo y desarrollo personal y profesional y facilitan su integración a la sociedad actual, en el caso de las mujeres, el acceso al conocimiento y la participación activa en los espacios de la sociedad de la información se convierte en una posibilidad para visibilizar su papel en el ámbito empresarial, acceder a nuevos trabajos y profesiones, desarrollar proyectos emprendedores para empoderar y mejorar sus vidas, favorecer su autonomía y salir del estado de vulnerabilidad en que se encuentran, minimizando los efectos de la denominada brecha digital de género.

\section{Referencias}

Aguilar, F. (2011). Reflexiones filosóficas sobre la tecnología y sus nuevos escenarios. Sophia, Colección de Filosofía de la Educación(11), 123-174. Obtenido de https://www.redalyc.org/pdf/4418/441846104007.pdf

Aguinaga, J. (2016). El uso de las TIC. Su influencia en los cambios individuales y sociales. Jóvenes $e$ identidades(1), 9-25. Obtenido de http://www.injuve.es/sites/default/files/revista111_ca p1.pdf
Alva, A. (2015). Los nuevos rostros de la desigualdad en el siglo xxi: la brecha digital. Revista Mexicana de Ciencias Políticas y Sociales, LX(223), 265-286. Obtenido de Dialnet-

LosNuevosRostrosDeLaDesigualdadEnElSigloXXI4933568.pdf

Arenas, M. (2011). Brecha digital de género : la mujer y las nuevas tecnologías. Anuario Facultad de DerechoUniversidad de Alcalá, IV, 97-125. Obtenido de https://ebuah.uah.es/dspace/bitstream/handle/10017/9 843/brecha_arenas_AFDUA_2011.pdf?sequence $=1$ \&isAllowed=y

Barrio, M., \& Manyoma, E. (2019). Mujer y Educación Superior en el Caribe Colombiano. En REOALCEI, La investigación universitaria en red en América Latina y sus differentes perspectivs (págs. 44-77). Cartagena de Indias, Colombia: Tecnológico de Comfenalco.

Bonder, G. (2002). Las nuevas tecnologías de información y las mujeres: reflexiones necesarias. Santiago de Chile: CEPAL. Obtenido

de https://www.cepal.org/es/publicaciones/5894nuevas-tecnologias-informacion-mujeresreflexiones-necesarias

Cabrera, P. (2005). Nuevas Tecnologías y exclusión social. Un estudio sobre las posibilidades de las TIC en la lucha por la inclusión en España. Madrid: UNESCO. Obtenido de https://www.ohchr.org/Documents/Issues/CulturalRi ghts/ConsultationEnjoyBenefits/UNESCONUEVAS _TECNOLOGIASyExclusionsocial.pdf

Castaño, C. (2009). La segunda brecha digital y las mujeres jóvenes. Cuadernos del Mediterráneo(11), 218-224. Obtenido de https://www.iemed.org/publicacions/quaderns/11/41 _la_segunda_brecha_digital.pdf

GEM. (2015). Informe GEM. España 2015. Obtenido de http://www.gem-spain.com/wpcontent/uploads/2015/03/Informe-GEM-2015esafp.pdf

Gil-Juarez, A., Vitores, A., Feliu, J., \& Vall-Llovera, M. (2011). Brecha digital de género: Una revisión y una propuesta. Education In The Knowledge Society (EKS), 12(2), 25-53. Obtenido de http://revistas.usal.es/index.php/eks/article/view/827 2

Lera, F., Hernández, N., \& Blanco, C. (2003). La "Brecha Digital" un reto para el desarrollo de la Sociedad del Conocimiento. Revista de la Economía Mundial(8), 119-142. Obtenido de https://www.semwes.org/sites/default/files/revistas/rem8_6.pdf

Mendoza, J., \& Caldera, J. (Mayo-Agosto de 2014). Umbrales para la determinación de la brecha digital:. TransInformação, 26(2), 125-132. Obtenido de http://www.scielo.br/pdf/tinf/v26n2/0103-3786-tinf26-02-00125.pdf

OCDE. (2008). Gender and Sustainable Development: Maximising the economic, social and environmental role of women. París: OECD. Obtenido de https://www.oecd.org/social/40881538.pdf

OIT. (2019). Competencias y Emprendimiento: reducir la brecha tecnológica y las desigualdades de género. Obtenido de 
https://www.ilo.org/wcmsp5/groups/public/@dgrepo rts/@gender/documents/publication/wcms_101145.p df

Romero, S., \& Varela, J. (2018). Mujer \& Tecnología 2018. UGT.

http://www.ugt.es/sites/default/files/migration/23-

04\%20MUJER\%20Y\%20TECNOLOGIA\%202018 $\% 20 \% 28$ ok\%29.pdf

Toudert, D. (2019). . Brecha digital, uso frecuente y aprovechamiento de Internet en México. Convergencia Revista de Ciencias Sociales(79), 1-27. Obtenido

https://convergencia.uaemex.mx/article/view/10332

Varela, J. (2015). La brecha digital en España. Estudio sobre la desigualdad postergada. Madrid: UGT. Obtenido de http://portal.ugt.org/Brecha_Digital/BRECHADIGIT AL_WEB.pdf

Viadana, C., Zubeldía, M., Tomarelli, J., \& Morales, O. (Noviembre 2016). Mujeres emprendedoras: una aproximación empírica. (págs. 2-18). Rosario, Argentina: UNR. Obtenido de https://www.fcecon.unr.edu.ar/webnueva/sites/default/files/u16/Decimocuartas/viadana_ zubeldia_y_otros_mujeres_emprendedoras.pdf 